\title{
Research Article \\ On Exact Controllability of First-Order Impulsive Differential Equations
}

\author{
Juan J. Nieto ${ }^{1}$ and Christopher C. Tisdell ${ }^{2}$ \\ ${ }^{1}$ Departamento de Análisis Matemático, Facultad de Matemáticas, Universidad de Santiago de Compostela, \\ Santiago de Compostela 15782, Spain \\ ${ }^{2}$ School of Mathematics and Statistics, The University of New South Wales, Sydney 2052, Australia
}

Correspondence should be addressed to Juan J. Nieto, juanjose.nieto.roig@usc.es

Received 5 October 2009; Revised 8 January 2010; Accepted 24 February 2010

Academic Editor: Josef Diblik

Copyright (C) 2010 J. J. Nieto and C. C. Tisdell. This is an open access article distributed under the Creative Commons Attribution License, which permits unrestricted use, distribution, and reproduction in any medium, provided the original work is properly cited.

\begin{abstract}
Many dynamical systems have an impulsive dynamical behavior due to abrupt changes at certain instants during the evolution process. The mathematical description of these phenomena leads to impulsive differential equations. In this work, we present some new results concerning the exact controllability of a nonlinear ordinary differential equation with impulses.
\end{abstract}

\section{Introduction}

Many evolution processes in nature are characterized by the fact that at certain moments in time they experience an abrupt change of state. Such behavior is seen in a range of problems from: mechanics; chemotherapy; population dynamics; optimal control; ecology; industrial robotics; biotechnology; spread of disease; harvesting; physics; medical models. The reader is referred to [1-8] and references therein for some models and applications to the above areas.

The branch of modern, applied analysis known as "impulsive" differential equations furnishes a natural framework to mathematically describe the aforementioned jumping processes. Consequently, the area of impulsive differential equations has been developing at a rapid rate, with the wide applications significantly motivating a deeper theoretical study of the subject [9-11].

Impulsive control systems have been studied by several authors [12-18]. In [15] the problem of controlling a physical object through impacts is studied, called impulsive manipulation, which arises in a number of robotic applications. In [16] the authors investigated the optimal harvesting policy for an ecosystem with impulsive harvest. For some recent references on different control strategies, including impulsive control, we refer the reader to $[13,19-26]$ and the references therein. 
Now, let $T>0$ and $0=t_{0}<t_{1}<t_{2}<\cdots<t_{m-1}<t_{m}<t_{m+1}=T, J=[0, T]$, and $J^{\prime}=[0, T] \backslash\left\{t_{1}, t_{2}, \ldots, t_{m}\right\}$, and $\lambda$ is an $n \times n$ real matrix.

Consider the following impulsive control differential equation:

$$
\begin{gathered}
x^{\prime}(t)+\lambda x(t)=f(t, x(t))+[B u](t), \quad t \in[0, T], t \neq t_{j}, j=1,2, \ldots, m, \\
x\left(t_{j}^{+}\right)=x\left(t_{j}^{-}\right)+I_{j}\left(x\left(t_{j}\right)\right), \quad j=1,2, \ldots, m,
\end{gathered}
$$

where $f: J^{\prime} \times \mathbb{R}^{n} \rightarrow \mathbb{R}^{n}, B$ is an operator defined on a set of admissible controls $u$, and $I_{j}: \mathbb{R}^{n} \rightarrow \mathbb{R}^{n}, j=1,2, \ldots, m$.

As usual, $x\left(t_{1}^{-}\right):=\lim _{t \rightarrow t_{1}^{-}} x(t)$ and $x\left(t_{1}^{+}\right):=\lim _{t \rightarrow t_{1}^{+}} x(t)$.

Our purpose is to the system (1.1)-(1.2) from the initial state $x_{0}$ to a desired final state $x_{1}$ in the finite time $T$.

We say that system (1.1)-(1.2) is exactly controllable in the time $T>0$ if for any $x_{0}, x_{1}$ there exists a control $u$ such that a solution $x$ of (1.1)-(1.2) satisfies $x(0)=x_{0}, x(T)=x_{1}$. Of course, we specify below the space of solutions and controls.

The main idea of our approach is to transform the controllability problem to the existence of a fixed point of an appropriate nonlinear operator generated by the original problem. This approach is not new and has been used by some authors such as Bhat [27], Chang et al. [28, 29], Sakthivel et al. [30], and Tonkov [31].

\section{Some General Results on Exact Controllability}

Consider the following finite-dimensional linear system:

$$
x^{\prime}(t)=A x(t)+b u(t)
$$

where $A$ is an $n \times n$ matrix, and $b \in \mathbb{R}^{n}$.

This linear system is completely controllable if for any $x_{0}, x_{1} \in \mathbb{R}^{n}$ there exists a $T>0$ and a control function $u(t)$ defined for $t \in[0, T]$ such that the solution to (2.1) with initial condition $x(0)=x_{0}$ satisfies $x(T)=x_{1}$. It is well known [32] that the linear system (2.1) is completely controllable if and only if

$$
\operatorname{rank}\left[\begin{array}{lllll}
b & A b & A^{2} b & \cdots & A^{n-1} b
\end{array}\right]=n .
$$

If the system is infinite dimensional, that is,

$$
x^{\prime}(t)=A x(t)+b u(t)
$$

where $A$ is the infinitesimal generator of a strongly continuous semigroup in a Hilbert space $X$, and $b: U \rightarrow X$ a linear bounded operator from a Hilbert space $U$ into $X$, then if the semigroup is compact the linear system (2.3) is not exactly controllable [33-35]. (1.1)-(1.2).

In this paper we study the finite-dimensional nonlinear impulsive control problem 


\section{Exact Controllability without Impulses}

Consider (1.1) without impulses, that is,

$$
x^{\prime}(t)+\lambda x(t)=f(t, x(t))+[B u](t), \quad t \in[0, T],
$$

with the initial condition

$$
x(0)=x_{0} .
$$

Here, $f:[0, T] \times \mathbb{R}^{n} \rightarrow \mathbb{R}^{n}$ continuous and $B: C[0, T] \rightarrow C[0, T]$.

In what follows, $B u=u$, and hence if $x \in C^{1}[0, T]$ is a solution of the initial problem (3.1)-(3.2), then

$$
x(t)=e^{-\lambda t} x_{0}+\int_{0}^{t} e^{-\lambda(t-s)}[f(s, x(s))+u(s)] d s
$$

We can define the following operator defined by the right-hand side of (3.3):

$$
\begin{gathered}
\mathcal{F}: C[0, T] \times C[0, T] \longrightarrow C[0, T], \\
{[\mathcal{F}(x, u)](t)=e^{-\lambda t} x_{0}+\int_{0}^{t} e^{-\lambda(t-s)}[f(s, x(s))+u(s)] d s .}
\end{gathered}
$$

In what follows $B$ is the identity operator so that the control space is $U=\mathbb{R}^{n}$. Note that $\mathcal{F}=\mathcal{F}_{x_{0}}$ depends on the initial condition $x_{0}$ and for any control

$$
[F(x, u)](0)=x_{0} .
$$

Now, for $x_{1} \in \mathbb{R}^{n}$, suppose that we are able to find a control $u=\mathcal{G}(x)$ such that

$$
[\mathcal{F}(x, \mathcal{G}(x))](0)=x_{0}, \quad[\mathcal{F}(x, \mathcal{G}(x))](T)=x_{1} .
$$

This means that for the control $u=\mathcal{G}(x)$, the system transfers the initial state to the desired final state if

$$
\mathscr{H}=\mathscr{F}(\cdot, \mathcal{G}(\cdot))
$$

has a fixed point. Consequently, if the operator $\mathscr{l}$, which of course depends on the initial state $x_{0}$, has a fixed point for any initial state, then the system is exactly controllable.

To clarify the ideas exposed above, suppose that $B u=u$ and let us introduce the control

$$
u(t)=\frac{1}{T} e^{\lambda(T-t)}\left[x_{1}-e^{-\lambda T} x_{0}-\int_{0}^{T} e^{-\lambda(T-s)} f(s, x(s)) d s\right]
$$


Define $\mathcal{G}: C[0, T] \rightarrow C[0, T]$ by the right-hand side in(3.8)

$$
[\mathcal{G}(x)](t)=\frac{1}{T} e^{\lambda(T-t)}\left[x_{1}-e^{-\lambda T} x_{0}-\int_{0}^{T} e^{-\lambda(T-s)} f(s, x(s)) d s\right] .
$$

Thus, for this control,

$$
\begin{aligned}
{[\mathscr{\ell}(x)](T)=} & {[\mathcal{F}(x, \mathcal{G}(x))](T)=e^{-\lambda t} x_{0}+\int_{0}^{T} e^{-\lambda(T-s)}[f(s, x(s))+\mathcal{u}(s)] d s } \\
= & e^{-\lambda t} x_{0} \\
& +\int_{0}^{T} e^{-\lambda(T-s)}\left[f(s, x(s))+\frac{1}{T} e^{\lambda(T-t)}\left[x_{1}-e^{-\lambda T} x_{0}-\int_{0}^{T} e^{-\lambda(T-\tau)} f(\tau, x(\tau)) d \tau\right]\right] d s \\
= & x_{1} .
\end{aligned}
$$

We have thus the following result.

Theorem 3.1. If for any initial condition $x_{0}$ and final condition $x_{1}$ the operator

$$
\mathscr{H}: C[0, T] \longrightarrow C[0, T]
$$

has a fixed point, then system (3.1) with $B u=u$ is exactly controllable.

\section{Exact Controllability with Impulses}

As usual, see any of the references on impulsive differential equations, we consider the Banach space

$$
\begin{aligned}
P C\left([0, T], \mathbb{R}^{n}\right)=\{ & x:[0, T] \longrightarrow \mathbb{R}^{n}: x \in C\left(J^{\prime}, \mathbb{R}^{n}\right), \\
& \left.x \text { is left continuous at } t_{j} \text { and the right - hand limits } x\left(t_{j}^{+}\right) \text {exist }\right\}
\end{aligned}
$$

with the norm

$$
\|x\|_{P C}=\sup _{t \in[0, T]}\|x(t)\|
$$

Let $J_{j}=\left[t_{j-1}, t_{j}\right], j=1,2, \ldots, p$, and $x_{j}$ the restriction of $x$ to that subinterval $J_{j}$.

The space

$P C^{1}\left([0, T], \mathbb{R}^{n}\right)=\left\{x \in P C\left([0, T], \mathbb{R}^{n}\right): x_{j} \in C^{1}\left(J_{j}, \mathbb{R}^{n}\right)\right.$, and the limits $x^{\prime}\left(t_{j}^{-}\right), x^{\prime}\left(t_{j}^{+}\right)$exist $\}$ 
with the norm

$$
\|u\|_{P C^{1}}=\max \left\{\|x\|_{P C},\left\|x^{\prime}\right\|_{P C}\right\}
$$

is a Banach space.

Now consider the impulsive control differential equation

$$
\begin{gathered}
x^{\prime}(t)+\lambda x(t)=f(t, x(t))+u(t), \quad t \in[0, T], t \neq t_{j}, j=1,2, \ldots, m, \\
x\left(t_{j}^{+}\right)=x\left(t_{j}^{-}\right)+I_{j}\left(x\left(t_{j}\right)\right), \quad j=1,2, \ldots, m,
\end{gathered}
$$

where $f: J^{\prime} \times \mathbb{R}^{n} \rightarrow \mathbb{R}^{n}$ is continuous and there exist the limits

$$
\begin{gathered}
f\left(t_{j}^{+}, x\right)=\lim _{t \rightarrow t_{1}^{+}} f(t, x), \quad f\left(t_{j}^{-}, x\right)=\lim _{t \rightarrow t_{j}^{-}} f(t, x), \\
f\left(t_{j}, x\right)=f\left(t_{j}^{-}, x\right),
\end{gathered}
$$

$u \in P C[0, T]$ and $I_{j}: \mathbb{R}^{n} \rightarrow \mathbb{R}^{n}$ are continuous

By a solution of (4.5)-(4.6), and for continuous controls $u(\cdot)$, we mean a function $x \in$ $P C^{1}\left([0, T], \mathbb{R}^{n}\right)$ satisfying (4.5) for every $t \in J^{\prime}$ and the impulses (4.6). In the case that the control $u$ is, for example, in the space $L^{2}$ locally, the solution must satisfy (4.5) for almost every $t \in J_{j}$ for each $j=0,1, \ldots, p$ and the impulses indicated in (4.6).

Lemma 4.1. If $x \in P C^{1}\left([0, T], \mathbb{R}^{n}\right)$ is a solution of (4.5)-(4.6), then $x$ satisfies

$$
x(t)=e^{-\lambda t} x_{0}+\int_{0}^{t} e^{-\lambda(t-s)}[f(s, x(s))+u(s)] d s+\sum_{0<t_{j}<t} e^{-\lambda\left(T-t_{j}\right)} I_{j}\left(x\left(t_{j}\right)\right) .
$$

Reciprocally, if $x \in P C\left([0, T], \mathbb{R}^{n}\right)$ satisfies (4.8), then $x$ is a solution of (4.5)-(4.6).

See [2] for the proof.

Now, define the operators $F: P C\left([0, T], \mathbb{R}^{n}\right) \times P C\left([0, T], \mathbb{R}^{n}\right) \rightarrow P C\left([0, T], \mathbb{R}^{n}\right)$ by

$$
[\mathcal{F}(x, u)](t)=e^{-\lambda t} x_{0}+\int_{0}^{t} e^{-\lambda(t-s)}[f(s, x(s))+u(s)] d s+\sum_{0<t_{j}<t} e^{-\lambda\left(T-t_{j}\right)} I_{j}\left(x\left(t_{j}\right)\right),
$$

and $\mathcal{G}: P C\left([0, T], \mathbb{R}^{n}\right) \rightarrow P C\left([0, T], \mathbb{R}^{n}\right)$ by

$$
[\mathcal{G}(x)](t)=\frac{1}{T} e^{\lambda(T-t)}\left[x_{1}-e^{-\lambda T} x_{0}-\int_{0}^{T} e^{-\lambda(T-s)} f(s, x(s)) d s-\sum_{j=1}^{m} e^{-\lambda\left(T-t_{j}\right)} I_{j}\left(x\left(t_{j}\right)\right)\right] .
$$

As in the nonimpulsive case, this control $u=\mathcal{G} x$ steers the system for the initial state $x_{0}$ to the final state $x_{1}$ in the finite time $T>0$.

Consequently we have the following result. 
Theorem 4.2. If $\mathscr{t}=\mathscr{F}(\cdot, \mathcal{G}(\cdot)): P C[0, T] \rightarrow P C[0, T]$ has a fixed point for any initial condition $x_{0}$ and final condition $x_{1}$, then the impulsive system (4.5)-(4.6) is exactly controllable.

\section{Main Results}

Schauder fixed point theorem states that any continuous mapping of a nonempty convex subset of a normed space into a compact set of that normed space has a fixed point [36, Theorem 4.1.1]. One of the most useful consequences is Schaefer's theorem [36, Theorem 4.3.2].

Theorem 5.1. Let $E$ be a normed space with $\mathscr{d e}: E \rightarrow E$ a compact mapping. If the set

$$
S:=\{x \in E: x=\mu d(x) \text { for some } \mu \in[0,1)\}
$$

is bounded then th has at least one fixed-point.

The operators $\mathcal{F}$ and $\mathcal{G}$ are continuous and compact $[2,37]$. Consequently, $\mathscr{t}$ is also continuous and compact and we can apply Schaefer's theorem.

Theorem 5.2. Suppose that $f$ has a sublinear growth, that is, there exist constants $a, b \geq 0$ and $0<\alpha<1$ such that for every $t \in J, x \in R^{n}$

$$
|f(t, x)| \leq a+b|x|^{\alpha}
$$

Assume that the impulses have sublinear growth. For every $j=1,2, \ldots, m$, there exist $a_{j}, b_{j} \geq 0$ and $0<\alpha_{j}<1$ such that for every $x \in R^{n}$ one has

$$
\left|I_{j}(x)\right| \leq a_{j}+b_{j}|x|^{\alpha_{j}}
$$

then the operator de has a fixed point for any $x_{0}, x_{1}$ and the impulsive system (4.5)-(4.6) is exactly controllable.

Proof. Let $x \in P C\left([0, T], \mathbb{R}^{n}\right)$. Using (5.2) and (5.3), it is evident that for any $t \in[0, T]$

$$
|[\mathcal{G}(x)](t)| \leq c_{0}+c_{0}^{\prime}\|x\|^{\alpha}+\sum_{j=1}^{m} c_{j}\|x\|^{\alpha_{j}},
$$

where $c_{0}, c_{0}^{\prime}, c_{j}, j=1,2, \ldots, m$ are constants.

Also, there exist constants $d_{0}, d_{0}^{\prime}, d_{0}^{\prime \prime}, d_{j}, j=1,2, \ldots, m$ such that for any $t \in[0, T]$,

$$
|[\mathcal{F}(x, u)](t)| \leq d_{0}+d_{0}^{\prime}\|x\|^{\alpha}+d_{0}^{\prime \prime}\|u\|+\sum_{j=1}^{m} d_{j}\|x\|^{\alpha_{j}}
$$


Combining these two last inequalities we get

$$
|[\mathscr{H}(x)](t)| \leq e_{0}+e_{0}^{\prime}\|x\|^{\alpha}+\sum_{j=1}^{m} e_{j}\|x\|^{\alpha_{j}}, \quad t \in[0, T],
$$

for some constants $e_{0}, e_{0}^{\prime}, e_{j}, j=1,2, \ldots, m$. Consequently, for any $x \in P C\left([0, T], \mathbb{R}^{n}\right)$ we have

$$
\|\mathscr{\ell}(x)\| \leq e_{0}+e_{0}^{\prime}\|x\|^{\alpha}+\sum_{j=1}^{m} e_{j}\|x\|^{\alpha_{j}}
$$

If $x \in P C\left([0, T], \mathbb{R}^{n}\right)$ is a solution of the equation $x=\mu \mathscr{\ell}(x), \mu \in(0,1)$, then

$$
\|x\|=\|\mu \mathscr{H}(x)\| \leq\|\mathscr{H}(x)\| \leq e_{0}+e_{0}^{\prime}\|x\|^{\alpha}+\sum_{j=1}^{m} e_{j}\|x\|^{\alpha_{j}} .
$$

For $\theta>0$, define $\Psi(\theta)=\theta-e_{0}-e_{0}^{\prime} \theta^{\alpha}+\sum_{j=1}^{m} e_{j} \theta^{\alpha_{j}}$. Noting that $\alpha, \alpha_{j} \in[0,1)$, we see that $\lim _{\theta \rightarrow+\infty} \Psi(\theta)=+\infty$. Taking $\theta=\|x\|$ we deduce that the set $\{\theta>0: \Psi(\theta) \leq 0\}$ is a bounded set.

Hence all the possible solutions of the equation $x=\mu \mathscr{\ell}(x), \mu \in(0,1)$, are bounded "a priori." By Schaeffer's theorem, $\mathscr{t}$ has a fixed point, which is equivalent to the exact controllability of the impulsive system (4.5)-(4.6).

As a consequence we have the following.

Theorem 5.3. Assume that $f$ is bounded and the impulses $I_{j}, j=1,2, \ldots, m$ are also bounded. then the operator de has a fixed point for any $x_{0}, x_{1}$ and the impulsive system (4.5)-(4.6) is exactly controllable.

When the nonlinearity $f$ is bounded, (4.5) is not exactly controllable in general. Even in the linear case $f(t, x)=A x$ the equation is not exactly controllable in general; see condition (2.2). However, by adding adequate impulses we can control the equation and hence the system becomes exactly controllable.

Example 5.4. Let $M$ be an $n \times n$ real matrix. Consider the system

$$
x^{\prime}=M x-\lambda x+b u
$$

such that $A=M-\lambda I$ does not satisfy (2.2). Then, (5.9) is not completely controllable. However, by adding the impulse

$$
I_{1}\left(x\left(t_{1}\right)\right)=\sqrt{\left\|x\left(t_{1}\right)\right\|}
$$

for some $t_{1} \in(0, T)$, the impulsive system (5.9)-(5.10) is completely controllable. 


\section{Acknowledgments}

The research of C. C. Tisdell was supported by funding from The Australian Research Council's Discovery Projects (DP0450752). The research of J. J. Nieto was partially supported by Ministerio de Educación y Ciencia and FEDER, Project MTM2007 61724, and by Xunta de Galicia and FEDER, Project PGIDIT06PXIB207023PR.

\section{References}

[1] S. Gao, L. Chen, J. J. Nieto, and A. Torres, "Analysis of a delayed epidemic model with pulse vaccination and saturation incidence," Vaccine, vol. 24, no. 35-36, pp. 6037-6045, 2006.

[2] J. J. Nieto, "Basic theory for nonresonance impulsive periodic problems of first order," Journal of Mathematical Analysis and Applications, vol. 205, no. 2, pp. 423-433, 1997.

[3] J. J. Nieto and R. Rodríguez-López, "Periodic boundary value problem for non-Lipschitzian impulsive functional differential equations," Journal of Mathematical Analysis and Applications, vol. 318, no. 2, pp. 593-610, 2006.

[4] A. d'Onofrio, "On pulse vaccination strategy in the SIR epidemic model with vertical transmission," Applied Mathematics Letters, vol. 18, no. 7, pp. 729-732, 2005.

[5] S. H. Saker and J. O. Alzabut, "Periodic solutions, global attractivity and oscillation of an impulsive delay host-macroparasite model," Mathematical and Computer Modelling, vol. 45, no. 5-6, pp. 531-543, 2007.

[6] A. M. Samoǔlenko and N. A. Perestyuk, Impulsive Differential Equations, vol. 14 of World Scientific Series on Nonlinear Science. Series A: Monographs and Treatises, World Scientific, River Edge, NJ, USA, 1995, With a preface by Yu. A. Mitropol'skiı̌ and a supplement by S. I. Trofimchuk. Translated from the Russian by Y. Chapovsky.

[7] J. Yan, A. Zhao, and J. J. Nieto, "Existence and global attractivity of positive periodic solution of periodic single-species impulsive Lotka-Volterra systems," Mathematical and Computer Modelling, vol. 40, no. 5-6, pp. 509-518, 2004.

[8] S. T. Zavalishchin and A. N. Sesekin, Dynamic Impulse Systems. Theory and Applications, vol. 394 of Mathematics and Its Applications, Kluwer Academic Publishers, Dordrecht, The Netherlands, 1997.

[9] J. J. Nieto and D. O'Regan, "Variational approach to impulsive differential equations," Nonlinear Analysis: Real World Applications, vol. 10, no. 2, pp. 680-690, 2009.

[10] H. Zhang, L. Chen, and J. J. Nieto, "A delayed epidemic model with stage-structure and pulses for pest management strategy," Nonlinear Analysis: Real World Applications, vol. 9, no. 4, pp. 1714-1726, 2008.

[11] H. Zhang, W. Xu, and L. Chen, "A impulsive infective transmission SI model for pest control," Mathematical Methods in the Applied Sciences, vol. 30, no. 10, pp. 1169-1184, 2007.

[12] R. K. George, A. K. Nandakumaran, and A. Arapostathis, "A note on controllability of impulsive systems," Journal of Mathematical Analysis and Applications, vol. 241, no. 2, pp. 276-283, 2000.

[13] G. Jiang and Q. Lu, "The dynamics of a prey-predator model with impulsive state feedback control," Discrete and Continuous Dynamical Systems. Series B, vol. 6, no. 6, pp. 1301-1320, 2006.

[14] X. Liu and A. R. Willms, "Impulsive controllability of linear dynamical systems with applications to maneuvers of spacecraft," Mathematical Problems in Engineering, vol. 2, no. 4, pp. 277-299, 1996.

[15] M. W. Spong, "Impact controllability of an air hockey puck," Systems \& Control Letters, vol. 42, no. 5, pp. 333-345, 2001.

[16] Y. Xiao, D. Cheng, and H. Qin, “Optimal impulsive control in periodic ecosystem," Systems E Control Letters, vol. 55, no. 7, pp. 558-565, 2006.

[17] Z. Yan, "Geometric analysis of impulse controllability for descriptor system," Systems $\mathcal{E}$ Control Letters, vol. 56, no. 1, pp. 1-6, 2007.

[18] J. Yao, Z.-H. Guan, G. Chen, and D. W. C. Ho, "Stability, robust stabilization and $H_{\infty}$ control of singular-impulsive systems via switching control," Systems E Control Letters, vol. 55, no. 11, pp. 879886, 2006.

[19] G. Agranovich, E. Litsyn, and A. Slavova, "Impulsive control of a hysteresis cellular neural network model," Nonlinear Analysis: Hybrid Systems, vol. 3, no. 1, pp. 65-73, 2009.

[20] A. Bressan, "Impulsive control of Lagrangian systems and locomotion in fluids," Discrete and Continuous Dynamical Systems. Series A, vol. 20, no. 1, pp. 1-35, 2008. 
[21] P. Georgescu and G. Moroşanu, "Pest regulation by means of impulsive controls," Applied Mathematics and Computation, vol. 190, no. 1, pp. 790-803, 2007.

[22] P. E. Kloeden and A. Rößler, "Runge-Kutta methods for affinely controlled nonlinear systems," Journal of Computational and Applied Mathematics, vol. 205, no. 2, pp. 957-968, 2007.

[23] J. Palczewski and L. Stettner, "Impulsive control of portfolios," Applied Mathematics and Optimization, vol. 56, no. 1, pp. 67-103, 2007.

[24] L. Run-Zi, "Impulsive control and synchronization of a new chaotic system," Physics Letters A, vol. 372, no. 5, pp. 648-653, 2008.

[25] Z. Yang and D. Xu, "Stability analysis and design of impulsive control systems with time delay," IEEE Transactions on Automatic Control, vol. 52, no. 8, pp. 1448-1454, 2007.

[26] H. Zhang, Z.-H. Guan, and G. Feng, "Reliable dissipative control for stochastic impulsive systems," Automatica, vol. 44, no. 4, pp. 1004-1010, 2008.

[27] S. P. Bhat, "Controllability of nonlinear time-varying systems: applications to spacecraft attitude control using magnetic actuation," IEEE Transactions on Automatic Control, vol. 50, no. 11, pp. 17251735, 2005.

[28] Y.-K. Chang, J. J. Nieto, and W. S. Li, "Controllability of semilinear differential systems with nonlocal initial conditions in Banach spaces," Journal of Optimization Theory and Applications, vol. 142, no. 2, pp. 267-273, 2009.

[29] Y.-K. Chang, W.-T. Li, and J. J. Nieto, "Controllability of evolution differential inclusions in Banach spaces," Nonlinear Analysis: Theory, Methods E Applications, vol. 67, no. 2, pp. 623-632, 2007.

[30] R. Sakthivel, N. I. Mahmudov, J. J. Nieto, and J. H. Kim, “On controllability of nonlinear impulsive integrodifferential systems," Dynamics of Continuous, Discrete E Impulsive Systems. Series A, vol. 15, no. 3, pp. 333-343, 2008.

[31] E. L. Tonkov, "Controllability of a nonlinear system in a linear approximation," Journal of Applied Mathematics and Mechanics, vol. 38, no. 4, pp. 557-565, 1974 (Russian).

[32] E. D. Sontag, Mathematical Control Theory: Deterministic Finite Dimensional Systems, vol. 6 of Texts in Applied Mathematics, Springer, New York, NY, USA, 2nd edition, 1998.

[33] J. P. Dauer and N. I. Mahmudov, "Approximate controllability of semilinear functional equations in Hilbert spaces," Journal of Mathematical Analysis and Applications, vol. 273, no. 2, pp. 310-327, 2002.

[34] R. Triggiani, "A note on the lack of exact controllability for mild solutions in Banach spaces," SIAM Journal on Control and Optimization, vol. 15, no. 3, pp. 407-411, 1977.

[35] R. Triggiani, "Addendum: "A note on the lack of exact controllability for mild solutions in Banach spaces"," SIAM Journal on Control and Optimization, vol. 18, no. 1, pp. 98-99, 1980.

[36] D. R. Smart, Fixed Point Theorems, Cambridge Tracts in Mathematics, no. 6, Cambridge University Press, London, UK, 1974.

[37] J. Li, J. J. Nieto, and J. Shen, "Impulsive periodic boundary value problems of first-order differential equations," Journal of Mathematical Analysis and Applications, vol. 325, no. 1, pp. 226-236, 2007. 